\title{
Amplitude Analysis of Lower Leg Muscle in Car Pedal Operation in Specific Driving Posture and Duration
}

\author{
Khamis, N.K. ${ }^{l}$ and Deros, B.M ${ }^{2}$ \\ ${ }^{1}$ Center for Materials Engineering and Smart Manufacturing (MERCU) \\ ${ }^{2}$ Center for Integrated Design for Advanced Mechanical System (PRISMA) \\ Faculty of Engineering and Built Environment, \\ Universiti Kebangsaan Malaysia, 43600 Bangi, Selangor, Malaysia.
}

\begin{abstract}
Driving posture is important to determine the comfortability of the driver. The purpose of this study is to investigate the muscle response in car pedal operation. To evaluate this response, 11 volunteers involved in simulated driving experiment using an electrical impedance myography to detect the muscle contraction of the lower leg, particularly at Gastrocnemius medial (GM). The driver requires to perform different pedal actions. The results depict that GM muscle shows different reaction according to pedal actions. According to these results, different degree of ankle angle lead to different muscle response. These findings help us to understand the effect of physical attribute related to muscle response and joint angle on driver during monotonous driving task.
\end{abstract}

\section{Key words: Driver, Car Pedal, Joint angle, Myography, Posture}

\section{INTRODUCTION}

Issues related to drivers are the subject of interest for many organisations such as road safety institutions, university researchers and also automotive manufacturers. Driver's comfortability is one of the main concern in ensuring safety driving experience. Interaction between driver and interior car components is a key to determine driver's comfort. In this case, this interaction can be defined as driving position or posture and reachability with the car control such as steering wheel, car pedal and gear transmission.

Up to this date, numerous research related to driver have been carried out by various parties around the world. Majority of the past studies focused on the driver's performance by using several methods, either objective or subjective assessment methods. Subjective methods are the most direct evaluation, due to the subjective perceptions regarding comfort or discomfort [1-2]. In contrast, objective methods require the use of specific equipment to measure the comfort condition. Nevertheless, objective methods produced more advantages compared to subjective methods, among which, it requires less time on observation and test subjects, less bias and measurement errors and can produce quick relevant information for the design process [3]. The objective methods are beneficial when they are integrated with subjective methods, provided there is a relationship between them [4].

Numerous studies conducted on interaction between driver and the car pedal. Based on the search engine from Science Direct by using driver and car pedal as the keywords, approximately 3700 results are found from 1995 to 2019. However, nearly 900 articles considering only relevant journals related to transportation, safety and ergonomics are produced in Science Direct website. As for example, four articles investigated a driver's behavior and performance when involving in pre-crash. [5-8]. Hault-Dubrelle et al. and Behr et al. for example, investigated the braking control system while operating the car [5-6].

Hault-Dubrelle et al. [6] analysed the driver motion based on their seating position by using 76 
volunteers. This study was conducted by using simulator. The volunteer positioning was recorded via five video cameras consisting of view from the front scene, the driver face, feet and pedals, hands on the steering wheel and global lateral view. Objective assessment methods by using braking force, seat pressure, and muscle measurement were collected in this study.

Behr et al. [5] concluded that a standard emergency braking configuration can be characterised according to joint angles, brake pedal load and muscular activation. Referring to this study, joint angle and muscle response are among important parameters to determine interaction between driver and car pedal.

Therefore, the purpose of the present study was to investigate the muscle reaction in three different pedal actions under simulated condition. The accelerator pedal was the key component in this study. This was done by using myography equipment to record the electrical impedance signal at the lower leg muscle. It was decided to conduct experiments with volunteers driving a car simulator in the laboratory. There are two hypotheses in this study:

i) Comparison between actions:

$\mathrm{H}_{0}$ : There is no significant differences between each car pedal action for selected muscle.

$\mathrm{H}_{1}$ : There is significant differences between each car pedal action for selected muscle.

ii) Comparison between pre and post activities:

$\mathrm{H}_{0}$ : There is no significant differences between pre and post activities in car pedal operation.

$\mathrm{H}_{1}$ : There is significant differences between pre and post activities in car pedal operation.

\section{METHODOLOGY}

\section{Simulator experiments}

Experiments on a car simulator were performed consisting of eleven volunteers (mean age of 29 years, mean height $=161 \mathrm{~cm}$, mean weight $=56 \mathrm{~kg}$ ). Each participant was required to attend one session, with instruction to wear flat sole shoes in the experiment. It is because this experiment is related to lower leg body part. In addition, the ankle angle when interacting with accelerator pedal was measured during experiment Therefore, this instruction should be fulfilled to determine accurate and reliable value for measurement. All volunteer were allowed to adapt with the car simulator setup and car seat adjustment before starting the experiment. The experiment was started after five minutes the participant had been in the driving position to allow them to adapt with the seat environment and fabrics. Prior to the actual experiment, all volunteer is first informed of the driving protocol.

The volunteer should sit on the car seat with the closest seated position to the car controls (mean knee angle $101.77^{\circ}$ ). The back rest of the car seat was fixed at $100^{\circ}$ in this study, as depicted in Figure 1. During recording the electrical impedance myography data as one of the objective assessment method in this study, the volunteer must place their hand at 10 and 2 o'clock as shown in Figure 1. In addition, the participant need to ensure the right leg at the car pedal, while the left leg at the car simulator floor, near to clutch pedal.

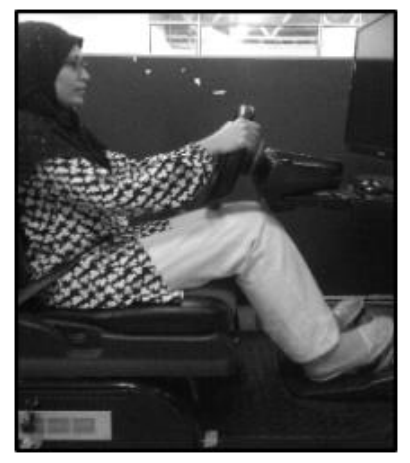

Fig 1. Driving position

The volunteer was required to obey to road rule as instructed by the researcher. Each volunteer is required to drive for 15 minutes with the driving task was reduced to a lane keeping task to induce task monotony. The simulated driving task was designed with the following requirements:

i) The volunteer should control the speed between 50 to $70 \mathrm{~km} / \mathrm{h}$ only.

ii) The volunteer was required to change the gear only at the beginning and at the end of the driving task.

iii) The driver cannot move their body from the car seat. They need to maintain their posture as instructed by the researcher. 
iv) The route was simple so that the drivers could complete the task as easily as possible.

v) There were few scenery changes.

vi) There was no inclination on driving route to reduce outside stimuli, and light curvature was chosen so that drivers should pay attention.

Even there is minimal contact of the car controls, particularly in gearing and braking system, there are other factors that will influenced driver's condition such as, car seat's pressure, driving duration, driving style, driving position and driver's charateristics. In fact, these factors are in agreement with the statements by other reseachers [9-13].

In this study, three main actions of the accelerator pedal (half press $=\mathrm{HP}$, release $=\mathrm{R}$ and full press $=F P$ ) were recorded at pre and post activities. Pre and post for EMG measurement was carried out before and after driving task. Roughly, five seconds is taken for each pedal action. This study is conducted to investigate the muscle response while performing these three different actions. Paired T-test was conducted to investigate difference between two parameters; i) actions (HP, R and FP) and ii) pre and post activities for all actions.

\section{Electrical impedance myography analysis}

Right gastrocnemius medial (GM) was evaluated for this study by using Electrical impedance myography equipment or known an electromyography (EMG). Trigno sensor was used to detect muscle signal in this study by using sample rate at $1000 \mathrm{~Hz}$. GM muscle was chosen based on literature studies for lower leg implication in driving [14-15]. Before placing the electrode, the belly of the muscle skin was identified by using certain activity as specified by Surface Electromyography for Non-Invasive Measurement (SENIAM) recommendations. The belly of muscle was shaved and cleaned by Nu-prep to prevent impedance mismatch and movement artifacts.

The EMG raw data was filtered by using Notch and band pass filter. Fifty Hertz is the setup for the Notch filter. This frequency was used to reduce the noise signal from any electrical device such as computer or hand phone that had been used near to the field work location. Band pass filter or known as analog filter is applied to the raw signal before it is digitized. This filter helps to remove low and high frequencies from the signal. There are two types of band filter, high pass and low pass. High band pass filter eliminates high frequency noise and prevents aliasing from occurring in the raw signal. In this study, the high pass value is $10 \mathrm{~Hz}$ cutoff. Ten Hertz was setup as cut off frequencies at the high-pass to ensure the signal was adjusted to zero line first. As a result, it can reduce the unwanted noise and artefact.

Low band pass filter eliminates baseline drift due to movement, biological artefact, and removes any DC offset. Five hundred Hertz is the cutoff filter for this type of band. SENIAM recommendations for surface EMG: high pass with 10-20 Hz cutoff, lowpass "near $500 \mathrm{~Hz}$ " cutoff, in most cases. Smoothing process was also conducted on the raw data signals. Fig. 2 illustrates the raw data processing from data collection by using EMG equipment to muscle signal comparison between pedal actions.

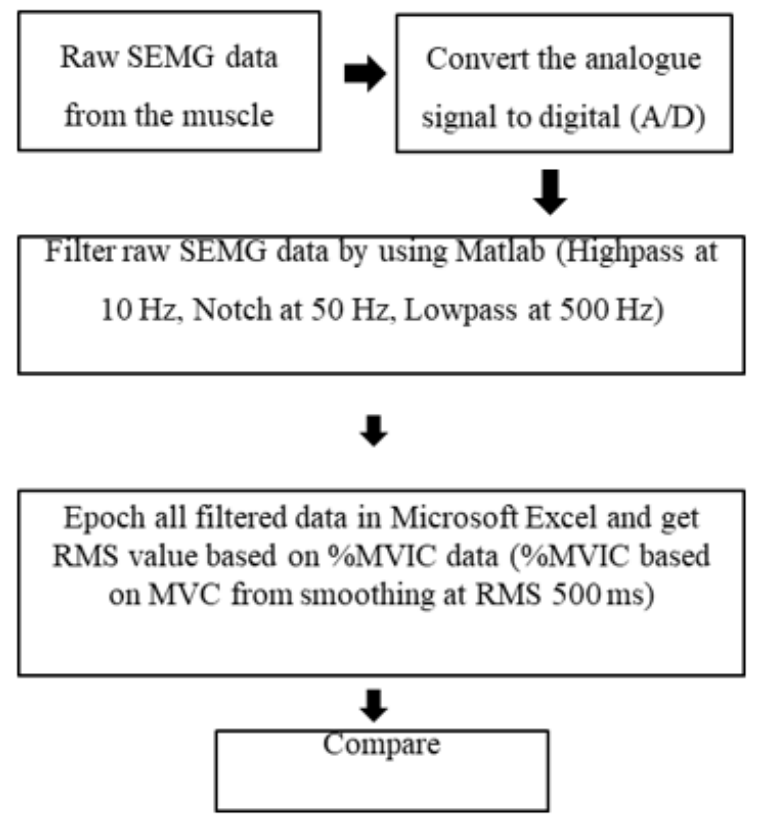

Fig 2. EMG data processing

After getting the cleaned data, Amplitude Analysis was carried out to determine muscle response in term of its contraction according to driving task. In this analysis, muscle contraction value can be determined in the form of Root Mean Square (RMS). If the RMS of muscle contraction below than 5 microvolt $(\mu \mathrm{V})$, it means the muscle is in the rest condition 
(Florimond 2009). Basically, the Amplitude Analysis was performed at time domain and the amplitude unit is in microvolt, $\mu \mathrm{V}$. The RMS equation in discrete time is defined in Equation (1), where $\mathrm{N}$ is the number of data and $\mathrm{n}$ is the SEMG data.

$$
\text { R.M.S }=\sqrt{\frac{1}{N} \sum_{n=1}^{n} E M G[n]^{2}}
$$

(Equation 1)

\section{RESULTS}

\section{Amplitude Analysis}

Figure 3 illustrates the amplitude analysis findings for the GM muscle. With respect to the difference between action, there is significant different pattern for all actions $(p<0.05)$. The mean RMS values of the accelerator pedal action at GM muscle increases with the ankle angle increment. In this case, based on the ankle angle, it showed that the toe when full pressing the accelerator pedal was in the flexion at mean $104^{0}(\mathrm{SD}=4.00)$. For releasing action, the ankle angle was at mean $90^{\circ}$ $(\mathrm{SD}=2.00)$. Meanwhile, for HP action, the ankle angle was at mean $97^{\circ}(\mathrm{SD}=2.00)$.

According to difference between pre and post activities, there is significant different pattern between pre-post activities, particularly at HP and R actions. For HP action, the mean RMS values for pre-post activity are 5.54 and $6.04 \mu \mathrm{V}$ respectively. For $\mathrm{R}$ action, the mean RMS values are 4.61 and $5.35 \mu \mathrm{V}$. Meanwhile, for FP action, the values are 6.77 and $6.67 \mu \mathrm{V}$, respectively.

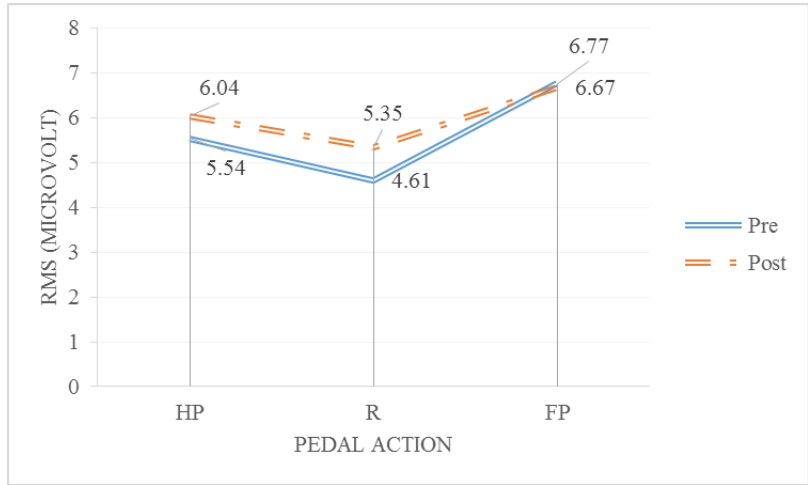

Fig 3. Amplitude analysis of GR for HP, R and FP actions according to pedal task

\section{DISCUSSION}

This study determined the lower leg muscle response while operating the accelerator pedal, particularly at GM muscle. There were three different actions when operating the accelerator pedal; HP, R and FP. According to the literature and supported with the findings in this study, the GM muscle plays important role when operating the pedal in driving task [14-15]. In this study, the highest muscle response at GM muscle was at FP action. In contrast, when releasing the pedal, the GM demonstrated the lowest activation which is nearly to $5 \mu \mathrm{V}$. According to these findings, it shows that the GM muscle is in the rest condition when releasing the pedal. According to Florimond [16], the muscle is in the rest condition when its amplitude is between $+/-5 \mu \mathrm{V}$. If more than $+/-5 \mu \mathrm{V}$, it is then in active form.

In addition, in terms of pre and post task, generally, there is no significant difference between both conditions for pedal control tasks. This finding shows that 15 minutes of driving activity does not really contribute to high muscle activation at the end of the journey. It is possibly due to the driving task in this study which do not require much accelerating and deaccelerating the pedal.

Table 1 and Table 2 shows the results based on through statistical analysis for responding to the two hypotheses for this study. As mentioned in earlier paragraph, there is significant difference between actions in accelerator pedal operation as tabulated in Table 1. Therefore, $\mathrm{H}_{0}$ is rejected and $\mathrm{H}_{1}$ is accepted. However, for second hypothesis, the $\mathrm{H}_{0}$ is accepted. Based on comprehensive analysis by using statistical method, there is no significant differences between pre and post activities for all actions as depicted in Table 2.

Table 1. Thorough statistical analysis (between actions)

\begin{tabular}{ccc}
\hline & Pre & Post \\
\hline HP-R & $\mathrm{Z}=-2.578$, & $\mathrm{Z}=-2.497$, \\
& $\mathrm{p}=0.010$ & $\mathrm{p}=0.013$ \\
FP-R & $\mathrm{Z}=-2.845$, & $\mathrm{Z}=-2.845$, \\
& $\mathrm{p}=0.004$ & $\mathrm{p}=0.004$ \\
HP-FP & $\mathrm{Z}=-2.848$, & $\mathrm{Z}=-2.713$, \\
& $\mathrm{p}=0.004$ & $\mathrm{p}=0.007$ \\
\hline
\end{tabular}


Table 2. Thorough statistical analysis (between pre and post activities)

\begin{tabular}{cccc}
\hline & $\mathrm{HP}$ & $\mathrm{R}$ & $\mathrm{FP}$ \\
\hline Pre-Post & $\mathrm{Z}=-0.255$. & $\mathrm{Z}=-1.599$ & $\mathrm{Z}=-0.459$ \\
& $\mathrm{p}=0.70$ & $\mathrm{p}=0.110$ & $\mathrm{p}=0.646$ \\
\hline
\end{tabular}

\section{CONCLUSION}

The leg placement while operating the accelerator pedal is expected to affect lower leg muscle activity. In this case, lower leg refers to calf, ankle and foot part. These body parts play important role when operating the pedal in driving task. In this study, muscle response of the GM was investigated by using EMG. Electrical signal from the selected muscle was collected and processed by using certain filter as explained in methodology section. This analysis was conducted in line with SENIAM recommendation for data processing.

Muscle response for GM was evaluated by using Amplitude Analysis. Based on this analysis, the findings demonstrate that different actions produce different muscle response $(p<0.05)$. In this study, releasing action depicted the lowest activation compared to pressing action for GM muscle. Furthermore, the GM muscle was in the rest condition when releasing the car pedal. The highest muscle response was in full pressing action. All in all, this study shows that the GM muscle works differently according to the car pedal actions. In term of pre and post activities, there is no significant difference between both periods based on comprehensive statistical analysis by using paired T-test ( $>0.05$ ).

In general, driving parameters play important role in determining driver's comfort. Driving position and driving duration are two controlled parameters in this study. The volunteer's driving position was restricted in term of its position between car control and car seat and also back rest inclination. Meanwhile, in term of driving duration, each volunteer is required to drive for 15 minutes with the driving task was reduced to a lane keeping task to induce task monotony. Based on this current study, further investigation was required to identify the relationship between varied driving parameters towards driver's comfort. For example, is is suggested to study the effect of close distant between car seat and car controls as well as extra duration of driving task on driver's comfort. These parameters certainly influence driver's comfort when operating the accelerator pedal.

\section{ACKNOWLEDGEMENT}

The authors acknowledge the financial support from Universiti Kebangsaan Malaysia under Grant GUP2017-094.

\section{REFERENCES}

[1] Khamis, N.K., Deros, B.M., Schramm, D., Hesse, B., Nuawi, M.Z. and Ismail, F.R., 2016. Subjective and indirect methods to observe driver's drowsiness and alertness: An overview. Journal of Engineering Science and Technology, 11, 28-39.

[2] Richards, L.G. 1980. On the psychology of passenger comfort. Human Factors in Transport Research, 2: 15-23.

[3] Lee, J., \& Ferraiuolo, P, 1993. Seat comfort. Technical Paper No. 931005.Society of Automotive Engineers, Inc., Warrendale, PA, USA

[4] De Looze, M.P., Kuijt-Evers, L.F.M., \& van Dieën, J. 2003. Sitting comfort and discomfort and the relationships with objective measures. Ergonomics, 46(10), 985-997.

[5] Behr, M., Poumarat, G., Serre, T., Arnoux, P.J., Thollon, L. and Brunet, C., 2010. Posture and muscular behaviour in emergency braking: An experimental approach. Accident Analysis \& Prevention, 42(3), 797801.

[6] Hault-Dubrulle, A., Robache, F., Pacaux, M.P. and Morvan, H., 2011. Determination of pre-impact occupant postures and analysis of consequences on injury outcome. Part I: A driving simulator study. Accident Analysis \& Prevention, 43(1), 66-74.

[7] Konstantopoulos, P., Chapman, P. and Crundall, D., 2010. Driver's visual attention as a function of driving experience and visibility. Using a driving simulator to explore drivers' eye movements in day, night and rain driving. Accident Analysis \& Prevention, 42(3), 827-834.

[8] Östh, J., Eliasson, E., Happee, R. and Brolin, K., 2014. A method to model anticipatory postural control 
in driver braking events. Gait \& posture, 40(4), 664669.

[9] Adler, S. 2007. The relation between long-term seating comfort and driver movement. Dipl.-Sportwiss, Friedrich-Schiller-Universitat Jena.

[10] Balasubramanian, V., \& Adalarasu, K. 2007. EMG-based analysis of change in muscle activity during simulated driving. Journal of Bodywork and Movement Therapies, 11(2), 151-158.

[11] Hiemstra-van Mastrigt, S., Groenesteijn, L., Vink, P. \& Kuijt-Evers, L.F., 2017. Predicting passenger seat comfort and discomfort on the basis of human, context and seat characteristics: a literature review. Ergonomics, 60(7), 889-911.

[12] Kyung, G., \& Nussbaum, M.A. 2008. Driver sitting comfort and discomfort (part II): Relationships with and prediction from interface pressure. International Journal of Industrial Ergonomics, 38(5-6), 526-538.
[13] Pandis, P., Prinold, J. A. I., \& Bull, A. M. J. 2015. Shoulder muscle forces during driving: sudden steering can load the rotator cuff beyond its repair limit. Clinical Biomechanics, 30(8), 839-846.

[14] Wang, X., Le Breton-Gadegbeku, B., \& Bouzon, L. 2004. Biomechanical evaluation of the comfort of automobile clutch pedal operation. International Journal of Industrial Ergonomics, 34(3), 209-221.

[15] Tanaka, Y., Kaneyuki, H., Tsuji, T., Miyazaki, T., Nishikawa, K., \& Nouzawa, T. 2009. Mechanical and perceptual analyses of human foot movements in pedal operation. Conference Proceedings - IEEE International Conference on Systems, Man and Cybernetics, (October), 1674-1679.

[16] Florimond, V., 2009. Basics of surface electromyography applied to physical rehabilitation and biomechanics. Montreal, Canada: Thought Technology Ltd. 